\title{
Rare Mechanism of Acquired Resistance to Osimertinib in Korean Patients with EGFR-Mutated Non-small Cell Lung Cancer
}

\author{
Jiyun Lee, MD ${ }^{1}$ \\ Joon Ho Shim, MD2,3 \\ Woong-Yang Park, MD, PhD $2,3,4$ \\ Hee Kyung Kim, MD, PhD' \\ Jong-Mu Sun, MD, PhD' \\ Se-Hoon Lee, MD, PhD' \\ Jin Seok Ahn, MD, PhD' \\ Keunchil Park, MD, PhD 1 \\ Myung-Ju Ahn, MD, PhD ${ }^{1}$
}

${ }^{1}$ Division of Hematology-Oncology, Department of Medicine, Samsung Medical Center, Sungkyunkwan University School of Medicine, Seoul, ${ }^{2}$ Samsung Genome Institute, Samsung Medical Center, Seoul, ${ }^{3}$ Department of Health Science and Technology, Samsung Advanced Institute for Health Sciences and Technology, Sungkyunkwan University, Seoul, ${ }^{4}$ Department of Molecular Cell Biology, Sungkyunkwan University School of Medicine, Seoul, Korea
Epidermal growth factor receptor (EGFR)-tyrosine kinase inhibitors (TKIs) are effective clinical therapeutics for EGFR-mutant non-small cell lung cancer (NSCLC). Osimertinib, a thirdgeneration EGFR TKI, has proven effective against T790M mutations. However, the vast majority of patients acquire resistance following successful treatment. A 59-year-old female patient with metastatic NSCLC developed resistance after 43 weeks of osimertinib. CancerSCAN of the metastatic liver lesion revealed a EGFR C797G mutation at an allele frequency of $72 \%$, a preexisting T790M mutation (73\%) in cis and an exon 19 deletion (87\%). Another 53-year-old female patient developed systemic progression after 10 months of osimertinib. CancerSCAN of the lung biopsy identified an EGFR L718Q mutation at an allele frequency of 7\%, concomitant PIK3CA E545K (12.90\%) and preexisting EGFR L858R (38\%), but loss of the T790M mutation. The heterogeneity of osimertinib resistance mechanisms warrants further investigation into novel or combination agents to overcome the rare acquired resistances.

Correspondence: Myung-Ju Ahn, MD, PhD

Division of Hematology-Oncology,

Department of Medicine, Samsung Medical

Center, Sungkyunkwan University School

of Medicine, 81 Irwon-ro, Gangnam-gu,

Seoul 06351, Korea

Tel: 82-2-3410-3459

Fax: 82-2-3410-1754

E-mail: silkahn@skku.edu

Received March 3, 2018

Accepted May 17, 2018

Published Online May 23, 2018

Key words

Osimertinib, AZD9291, Resistance

\section{Introduction}

East Asian never-smokers have a higher prevalence of epidermal growth factor receptor (EGFR) mutations compared to their North American/European counterparts [1]. EGFRtyrosine kinase inhibitors (TKIs) are effective clinical thera- pies for EGFR-mutant non-small cell lung cancer (NSCLC) patients [2]. Osimertinib, a third-generation EGFR TKI, has proven effective against T790M mutation. However, the vast majority of patients acquire resistance following successful treatment.

Many studies have reported that the EGFR C797S mutation is the most common mechanism of resistance to third-gener- 

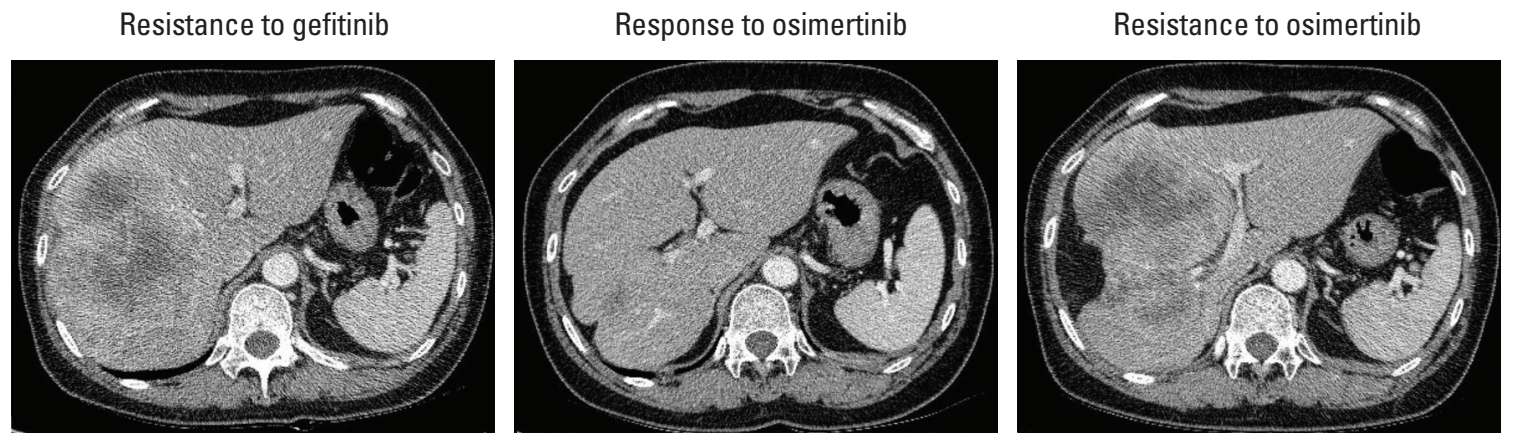

A

B
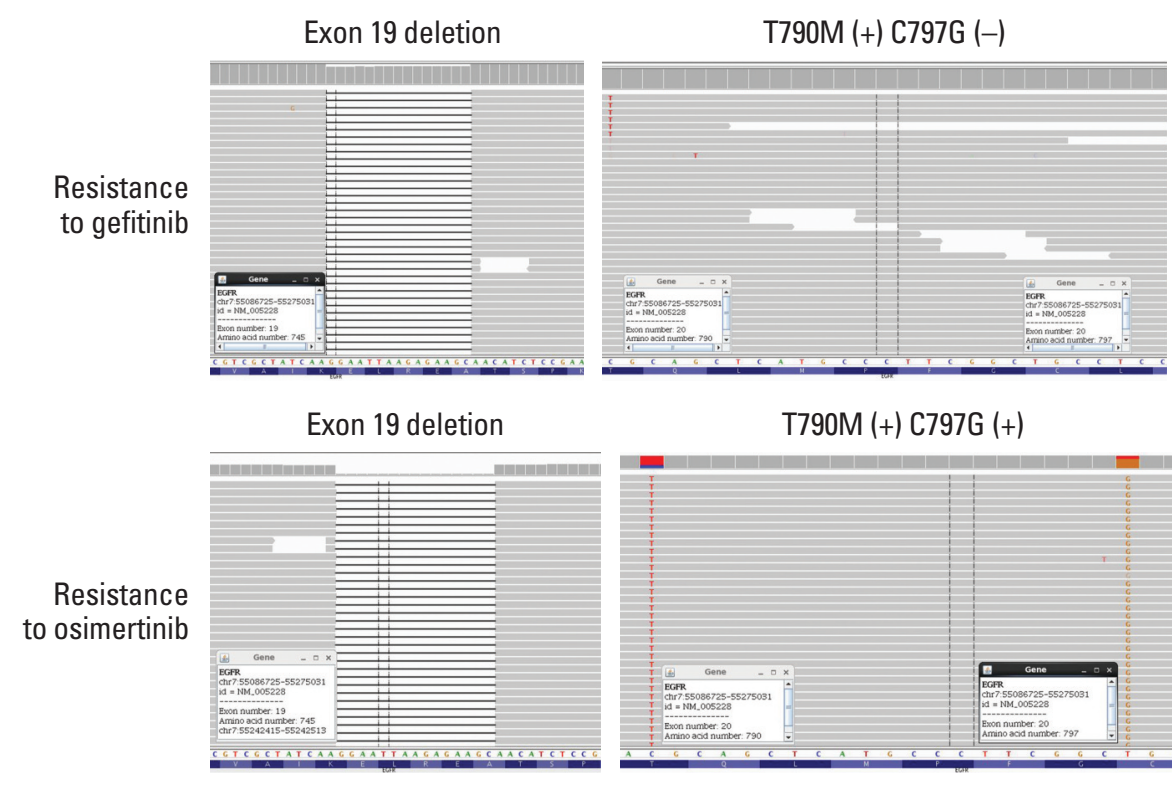

T790M (+) C797G (+)

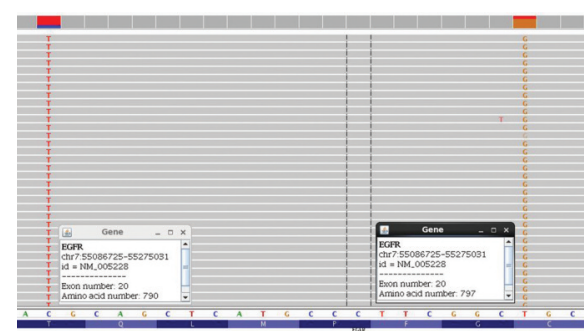

Fig. 1. Acquired epidermal growth factor receptor (EGFR) C797G mutation. (A) Computed tomography of the liver demonstrates the tumor at baseline before osimertinib treatment (left), at partial response (middle), and during progression (right) after treatment with osimertinib. (B) The browser view reveals the mutational status of the tumor at baseline (top) and after osimertinib therapy (bottom). (Continued to the next page)

ation EGFR TKIs, including osimertinib (AZD9291) [3,4]. Here we report two Korean cases of a rare mutation-EGFR C797G, L718Q_ as a mechanism of acquired resistance to osimertinib.

The medical records of these patients were retrospectively reviewed with the approval of the Institutional Review Board of the Samsung Medical center (IRB No. SMC 2018-05076) with the waiver for informed consent.

\section{Case Report}

\section{Case 1}

A 59-year-old female never-smoker was diagnosed with EGFR exon 19 deletion-positive metastatic lung adenocarcinoma, which was poorly differentiated with squamoid differentiation, in March 2015. The pathology result was initially reported as squamous cell carcinoma, and the patient underwent chemotherapy with four cycles of gemcitabine 

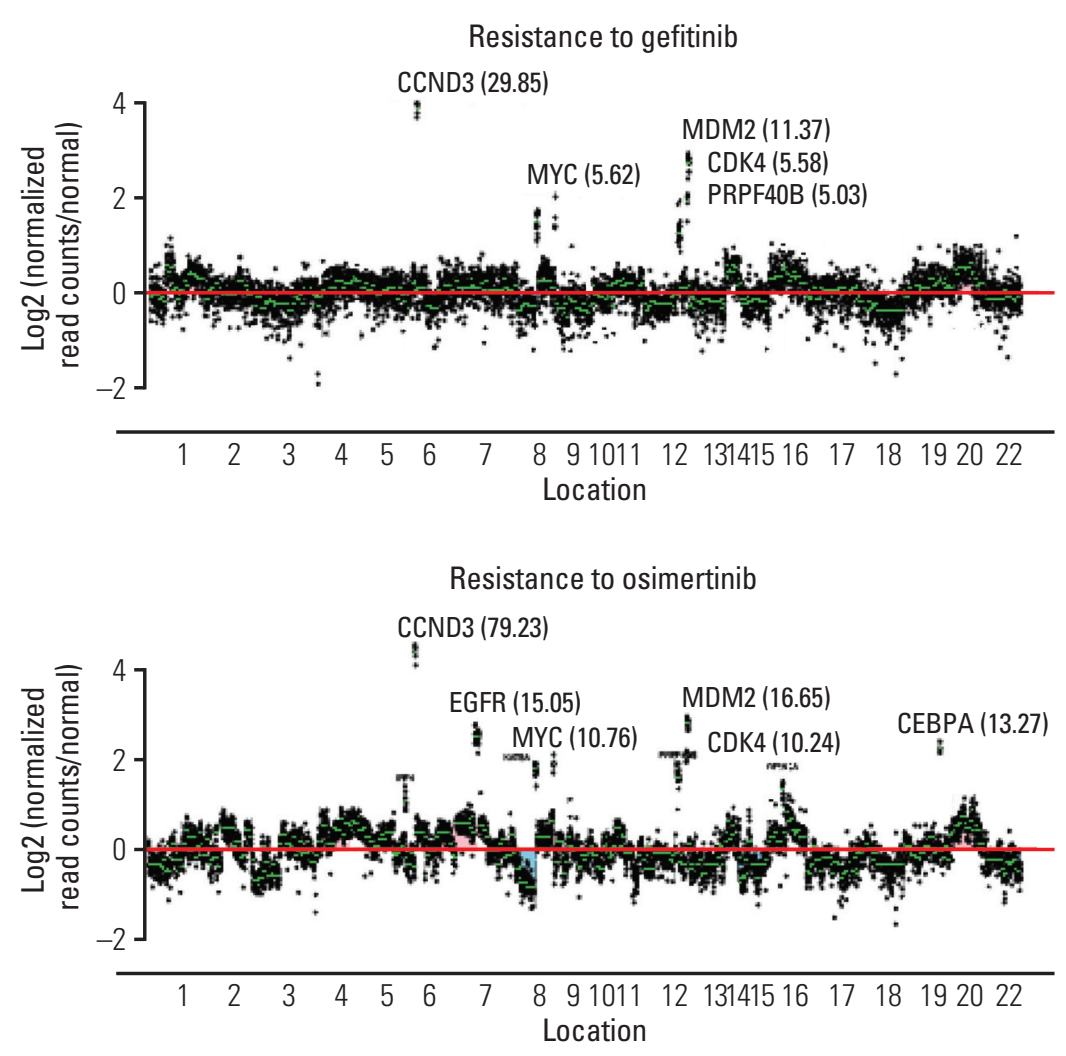

Fig. 1. (Continued from the previous page) (C) Plot depicting EGFR (15.0) copy number amplification upon treatment with osimertinib.

and carboplatin, leading to partial remission of the primary lesion in the left lower lobe. After seven months off treatment, the primary tumor in the left lower lobe, pleural seeding, and hepatic metastasis had increased in extent. The patient received gefitinib from January 2016 to December 2016, until progression of hepatic metastasis was demonstrated. Both real-time polymerase chain reaction (PCR) and CancerSCAN [5] of the post-gefinitib liver biopsy revealed an EGFR T790M mutation.

After 8 weeks of osimertinib treatment, scans demonstrated a partial response; however, the patient developed hepatic metastasis progression after 43 weeks of osimertinib (Fig. 1A). CancerSCAN [5] of the liver biopsy at the time of progression revealed an EGFR C797G mutation at an allele frequency of $72 \%$, including the known $\mathrm{T} 790 \mathrm{M}$ mutation $(73 \%)$ in cis and an exon 19 deletion $(87 \%)$, as well as focal amplification of EGFR (15.0) (Fig. 1B and C). As opposed to other preexisting N-ethyl-N-nitrosourea (ENU) mutagenesis studies, there was no coexisting EGFR C797S mutation. EGFR C797G mutation was the sole mutation with high minor allele frequency.

\section{Case 2}

A 53-year-old female never-smoker was diagnosed with advanced lung adenocarcinoma in October 2013. A pleural tumor specimen showed a missense mutation on exon 21, and the patient received gefitinib for 33 months as the first-line therapy. While continuing gefitinib, the patient underwent whole-brain irradiation for multiple brain metastases and leptomeningeal seeding. Progressive disease was detected, and a new pulmonary biopsy identified T790M mutation. The patient received 10 months of osimertinib from August 2016 to May 2017, until disease progression was confirmed (Fig. 2A).

At the time of systemic progression, cancerSCAN [5] was performed on the lung biopsy specimen and identified an EGFR L718Q mutation at an allele frequency of $7 \%$ and a preexisting EGFR L858R at an allele frequency of 38\%, but loss of the T790M mutation (Fig. 2B). Concomitant PIK3CA E545K at an allele frequency of $12.9 \%$ was also identified.

The patient received afatinib, the most effective EGFR inhibitor in cell line models harboring L858R along with 
Resistance to gefitinib

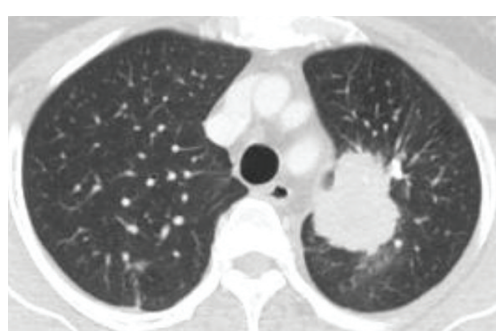

Response to osimertinib

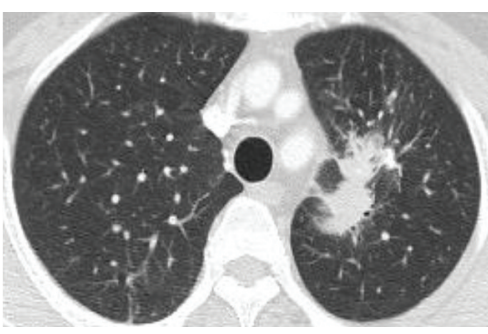

A

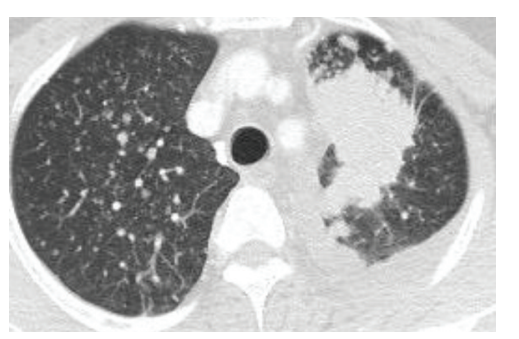

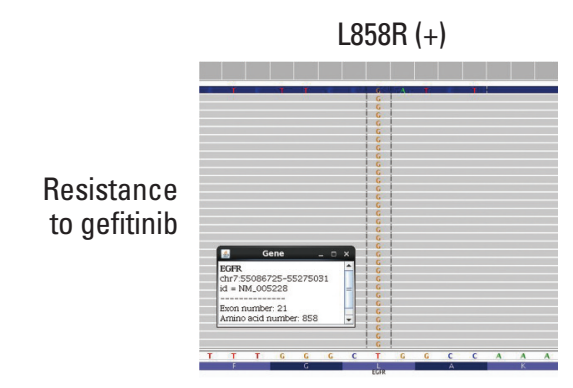

$\operatorname{L858R}(+)$

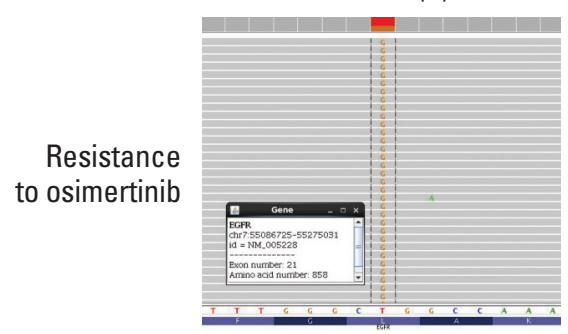

$\operatorname{T790M}(+)$

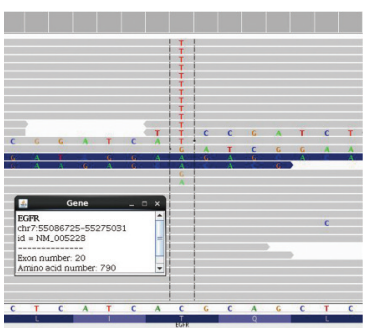

$\operatorname{T790M}(+)$

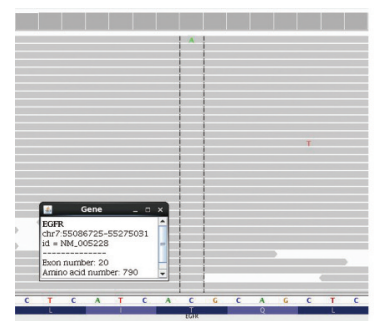

L7180 (-)

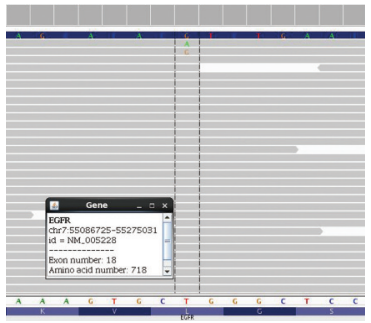

$\mathrm{L} 7180(+)$

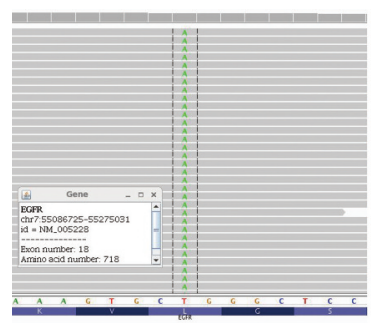

PIK3CA p.E545K (-)

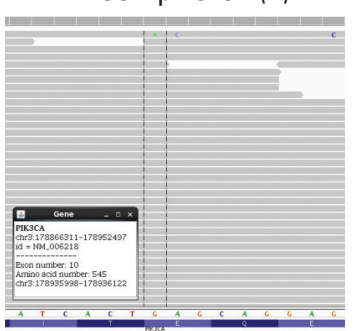

PIK3CA p.E545K (+)

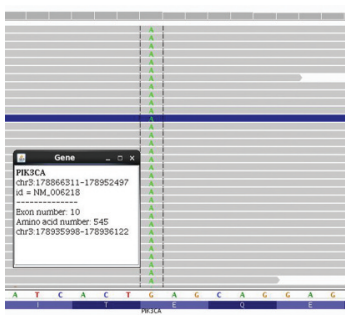

Fig. 2. Acquired epidermal growth factor receptor (EGFR) L718Q mutation. (A) Computed tomography of the chest showed the tumor at baseline before osimertinib treatment (left), at partial response (middle), and during disease progression (right) after treatment with osimertinib. (B) The browser view reveals the mutational status of the tumor at baseline (top) and after osimertinib therapy (bottom).

L718Q mutation [4], which induced partial remission after 4 weeks of therapy; however, she demonstrated systemic progression after 12 weeks of therapy.

\section{Discussion}

Herein, we describe two Korean patients with a rare EGFR mutation after cancer treatment with osimertinib, a thirdgeneration EGFR TKI.

The EGFR C797S mutation is detected in $~ 40 \%$ of EGFR- mutant NSCLC patients with T790M who develop acquired resistance to osimertinib [4]. However, emerging clinical data have revealed a high degree of clonal heterogeneity in the resistance mechanism, including non-EGFR-driven mutations.

Pre- and post-osimertinib genomic profiling results of cases 1 and 2 is presented in S1 and S2 Tables.

In case 1, although the variant allele frequency of EGFR T790M was detected pre-osimertinib CancerSCAN [5] at a low rate $(0.4 \%)$ (S1 Table), it was simultaneously detected on real-time PCR. In refractory tumors, a driver mutation that arises in response to treatment may be present with a low variant allele frequency [5]. Considering the durable res- 
ponse to osimertinib, EGFR T790M appears to be the most reasonable mechanism of resistance to gefitinib.

In case 2, pathway alterations beyond EGFR were reported from post-osimertinib CancerSCAN of the lung specimen, most notably PIK3CA E545K (12.90\%) (S2 Table).

Co-occurrence of the PIK3CA mutation with EGFR aberrations are frequently reported [6], and an in vitro study has shown that PIK3CA oncogenes are sufficient to promote continued phosphoinositide 3-kinase (PI3K) signaling in EGFRmutant cancer cells treated with EGFR TKIs [7]. Thus, cancer with PIK3CA oncogenic mutations exhibit attenuated responses to EGFR inhibitors, suggesting that these mutations the mechanism of acquired resistance [8]. Such acquired resistance possibly resulted in the short response duration observed with afatinib in case 2 .

Although EGFR mutation still remains the principal "mutational driver," there is increasing evidence of complexity in the resistance mechanism to third-generation EGFR TKIs. Dactolisib, a dual PI3K/mammalian target of rapamycin (mTOR) inhibitor, has proven effective in inhibiting gefintib-resistant tumor growth by downregulating PI3K/ anaplastic lymphoma kinase (ALK) / mTOR phosphorylation [9]. However, a phase II trial of MK-2206 (AKT inhibitor for PI3K/ ALK/mTOR pathway) and erlotinib in NSCLC patients who progressed on erlotinib, showed a frustrating Response Evaluation Criteria in Solid Tumor response rate $9 \%$ at 12 weeks in the EGFR-mutant group.

Our cases highlight the importance of a genomic profiling approach to detect alterations associated with uncommon mechanisms of acquired resistance to targeted therapies. Further studies are warranted on novel or combination agents to overcome the rare acquired resistances.

\section{Electronic Supplementary Material}

Supplementary materials are available at Cancer Research and Treatment website (https:// www.e-crt.org).

\section{Conflicts of Interest}

Conflict of interest relevant to this article was not reported.

\section{References}

1. Ohashi K, Maruvka YE, Michor F, Pao W. Epidermal growth factor receptor tyrosine kinase inhibitor-resistant disease. J Clin Oncol. 2013;31:1070-80.

2. Huang YH, Hsu KH, Tseng JS, Chen KC, Hsu CH, Su KY, et al. The association of acquired T790M mutation with clinical characteristics after resistance to first-line epidermal growth factor receptor tyrosine kinase inhibitor in lung adenocarcinoma. Cancer Res Treat. 2018;50:1294-303.

3. Wang S, Tsui ST, Liu C, Song Y, Liu D. EGFR C797S mutation mediates resistance to third-generation inhibitors in T790Mpositive non-small cell lung cancer. J Hematol Oncol. 2016; 9:59.

4. Ercan D, Choi HG, Yun CH, Capelletti M, Xie T, Eck MJ, et al. EGFR mutations and resistance to irreversible pyrimidinebased EGFR inhibitors. Clin Cancer Res. 2015;21:3913-23.

5. Shin HT, Choi YL, Yun JW, Kim NK, Kim SY, Jeon HJ, et al. Prevalence and detection of low-allele-fraction variants in clin- ical cancer samples. Nat Commun. 2017;8:1377.

6. Scheffler M, Bos M, Gardizi M, Konig K, Michels S, Fassunke $\mathrm{J}$, et al. PIK3CA mutations in non-small cell lung cancer (NSCLC): genetic heterogeneity, prognostic impact and incidence of prior malignancies. Oncotarget. 2015;6:1315-26.

7. Engelman JA, Mukohara T, Zejnullahu K, Lifshits E, Borras AM, Gale CM, et al. Allelic dilution obscures detection of a biologically significant resistance mutation in EGFR-amplified lung cancer. J Clin Invest. 2006;116:2695-706.

8. Wang J, Wang B, Chu H, Yao Y. Intrinsic resistance to EGFR tyrosine kinase inhibitors in advanced non-small-cell lung cancer with activating EGFR mutations. Onco Targets Ther. 2016; 9:3711-26.

9. Sun Z, Li Q, Zhang S, Chen J, Huang L, Ren J, et al. NVPBEZ235 overcomes gefitinib-acquired resistance by down-regulating PI3K/AKT/mTOR phosphorylation. Onco Targets Ther. 2015;8:269-77. 